\title{
Highly efficient plasmon-mediated electron injection into cerium oxide from embedded silver nanoparticles
} Received 00th January $20 \mathrm{xx}$
Accepted 00th January $20 \mathrm{xx}$ DOI: $10.1039 / \mathrm{x} 0 \times x 00000 \mathrm{x}$
Jacopo Stefano Pelli Cresi a,b, Maria Chiara Spadaro a,b, Sergio D`Addato a,b Sergio Valeri ${ }^{a, b}$, Stefania Benedetti ${ }^{a, b}$, Alessandro Di Bona ${ }^{b}$, Daniele Catone ${ }^{c}$, Lorenzo Di Marioc, Patrick O'Keeffe ${ }^{d}$, Alessandra Paladini $^{d}$, Giovanni Bertoni ${ }^{e}$, Paola Luches ${ }^{b^{*}}$

The coupling with plasmonic metal nanoparticles (NPs) represents a promising opportunity to sensitize wide band gap oxide to visible light. The processes which come into play after the excitation of localized surface plasmon resonances (LSPR) in the NPs largely determine the efficiency of the charge/energy transfer from the metal NP to the oxide. We report a study of plasmon mediated energy transfer from mass-selected silver NPs into the cerium oxide matrix in which they are embedded. Femtosecond transient absorption spectroscopy is used to probe the dynamics of charge carrier relaxation after the excitation of the LSPR of the silver nanoparticles and to evaluate the plasmon-mediated electron transfer efficiency from the silver nanoparticles to the cerium oxide. High injection efficiencies in the 6-16\% range have been identified for excitation between 400 and $600 \mathrm{~nm}$. These high values have been explained in terms of plasmon-mediated direct electron injection as well as indirect hot electron injection from the NPs to the oxide. The information obtained provide an important contribution towards a knowledge-driven design of efficient cerium oxide based nanostructured materials for solar to chemical energy conversion.

\section{Introduction}

The study of plasmon-mediated photocatalysis has increased very rapidly in recent years due to the possibility of extending the energy range of activity of traditional semiconducto photocatalysts, as in the case of the water splitting reaction induced by $\mathrm{TiO}_{2}$ sensitized by gold nanoparticles (NPs). ${ }^{1-7}$ This is generally achieved by taking advantage of the large cross section for the absorption and scattering of visible light of plasmonic metal nanostructures placed in the vicinity of a semiconductor substrate, thus creating a metal/semiconductor interface across which electrons can be transferred. ${ }^{8-10}$ In the class of semiconductor catalysts, cerium oxide $\mathrm{CeO}_{2}$ (the material studied here) is attracting a growing interest due to its efficiency in catalyzing redox reactions. ${ }^{11-14}$ In $\mathrm{CeO}_{2}$ the presence of localized Ce $4 \mathrm{f}$ states between the $\mathrm{O} 2 \mathrm{p}$ valence band and the Ce $5 d$ conduction band makes the material a very sensitive probe to identify charge transfer to/from neighboring metal atoms. ${ }^{15}$ The occupation of $4 \mathrm{f}$ levels is in turn expected to

- Dipartimento FIM, Università di Modena e Reggio Emilia, Via G. Campi 213/a Modena, Italy

di Ricerca S3, via G. Campi 213/a, Modena, Italy

C

CNR-ISM Division of Ultrafast Processes in Materials (FIASHit), Area dello Ricerca di Roma 1, Monterotondo Scalo, Italy

Istituto dei Materiali per l'Elettronica ed il Magnetismo, Consiglio Nazionale delle Ricerche, Parco Area delle Scienze 37/A, I-43124, Parma, Italy

* Corresponding Author e-mail: paola.luches@nano.crr.tt

Electronic Supplementary Information (ESI) available: (details of any supplementary

information available should be included here]. See DOI: 10.1039/x0xx00000x modify the material properties, inducing a decrease of oxygen vacancy formation energy, and a change of the optical response. ${ }^{16,17}$

A plethora of different mechanisms for the transfer of energy from metal NPs to semiconductors have been identified and recently reviewed. ${ }^{18-21}$ The dominant processes which come into play are: i) the enhancement of an optical transition within the semiconductor due to the presence of the plasmonic NPs, 6 ii) hot electron injection from the plasmonic NPs to the semiconductor, ${ }^{6}$ and the recently described iii) direct metal-tosemiconductor interfacial charge transfer. 22,23

The first of these is actually a group of mechanisms, which require an overlap between the localized surface plasmoh resonance (LSPR) of the NPs and the absorption band of the semiconductor and rely on the high light absorption cross section of the nanostructures to efficiently convert the energy of the light into electronic transitions within the semiconductor. These mechanisms include light trapping, by which we mean multiple and high angle elastic scattering of light by the NPs, leading to an increase in the effective optical path,18 energy transfer to the semiconductor through near-field enhancement in the vicinity of the NPs, 19 plasmon-induced radiative energy transfer in which photons emitted by the NPs are absorbed by the semiconductor, ${ }^{19}$ and plasmon-induced resonant energy transfer. ${ }^{24}$ In general, these processes contribute little to the efficiency of visible light energy transfer in the case of wide band gap semiconductors, which have low absorption cross sections in this region (e.g. $\mathrm{TiO}_{2}$ ). The second mechanism involves the formation of hot electrons in the metallic NPs either by interband transitions or decay of the LSPR by Landau ha eliminato: The main text of the article should appear here with headings as appropriate.

ha eliminato: the 
damping. The hot electrons can be transferred over/through the Schottky barrier formed at the interface into empty levels of the semiconductor. Finally, a mechanism has been proposed involving direct electron transfer during the excitation light pulse: the decay of the LSPR directly excites electrons into acceptor states of the semiconductor and leaves holes in the metal. 22,23 This process is possibly more efficient than the indirect transfer, where the hot electrons generated in the metal NP can be partially quenched by electron-electron and electron-phonon scattering before the injection. ${ }^{6}$

The relative contributions of the different mechanisms depend on numerous factors such as the shape, the density and the composition of the NPs, the band structure of the semiconductor, the morphology of the interface and many more. For example, an important factor to consider is the nearfield concentration of light due to the nanoantenna effect of the metallic nanostructures. ${ }^{25}$ It is well known that the spatia distribution of the electromagnetic field intensity is strongly dependent on the NPs shape and size and that it can be highly concentrated in specific sites, in particular in the case of interacting NPs with small interparticle distance or sharp edges. In the hot spots formed in this way the efficiency of the electron injection mechanism can be significantly modified. ${ }^{25-2}$ Moreover, it was shown that by embedding the metallic nanostructures into the semiconductor it is possible to significantly increase the injection efficiency due to a more extended metal-semiconductor contact area. 30,31

In this work we use femtosecond transient absorption spectroscopy (FTAS) to investigate the mechanisms of LSPR decay in silver NPs embedded in a thin film of $\mathrm{CeO}_{2}$. The samples are accurately controlled in stoichiometry and morphology, based on our previous experience with cerium oxide films in combination with $\mathrm{Ag}$ NPs. ${ }^{16,32}$ To maximize the $\mathrm{Ag} / \mathrm{CeO}_{2}$ interface and to prevent $\mathrm{Ag}$ oxidation the NPs are embedded within a cerium oxide matrix $\left(\mathrm{Ag} @ \mathrm{CeO}_{2}\right)$. We obtained very high electron injection efficiencies (6-16\%) that we discuss in terms of the above mechanisms, analyzing the role of the morphology of the metal NPs, of the metal/semiconductor interface, as well as the near-field light concentration effects due to hot-spot generation by the metal NPs.

\section{Methods}

The NPs were synthesized using a magnetron sputtering nanocluster source by inert gas aggregation (NC200U, Oxford Applied Research). The source chamber is part of an ultrahigh vacuum system that includes also an X-ray photoelectron spectroscopy (XPS) apparatus (a double anode $x$-ray source Specs XR50 coupled with a hemispherical electron analyzer Specs Phoibos 150) for in-situ chemical characterization. Silver NPs were obtained from a $99.9 \%$ pure $\mathrm{Ag}$ target $\left(P_{\text {shot }}=68 \mathrm{~W}\right)$ with an $\mathrm{Ar}$ gas flow $\left(\mathrm{f}_{\mathrm{Ag}}=59 \mathrm{sccm}\right)$ in the aggregation region and they were mass selected using a quadrupole mass filter (QMF200, Oxford Applied Research), set to select NPs with mass of $10^{6} \mathrm{amu}(\mathrm{F}=5 \mathrm{kHz}, \mathrm{V}=250 \mathrm{~V})$. The deposition chamber wassequipped with an electron bombardment evaporation cell, which was used to evaporate cerium oxide films using an oxygen partial pressure of $\mathrm{P}_{02}=5 \times 10^{-6} \mathrm{mbar}$. The chamber hosted a quartz crystal microbalance, which was used to estimate the evaporation rates from the nanocluster source and from the cerium evaporator before the growth. The sample under study was grown on a quartz substrate (fused silica EOX thickness $1 \mathrm{~mm}$ ) kept at room temperature by the sequential deposition of a cerium oxide film of $5 \mathrm{~nm}$ thickness, a layer of Ag NPs of $5 \mathrm{~nm}$ nominal thickness and a cerium oxide film of 5 $\mathrm{nm}$ thickness. The nominal thickness of the NP layer is defined as the thickness of a homogeneous film with the same volume as the NP layer. The two cerium oxide films were exposed to an oxygen partial pressure $\mathrm{P}_{\mathrm{O} 2}=6 \times 10^{-5} \mathrm{mbar}$ for $30 \mathrm{~min}$ after the growth, to maximize the $\mathrm{Ce}^{4+}$ concentration. After each growth step the sample was characterized in-situ by XPS using Al-Ka photons to obtain quantitative information on the amount of $\mathrm{CeO}_{2}$ and $\mathrm{Ag}$ deposited and on the oxidation state of $\mathrm{CeO}_{2}$ (see Supporting Information, Figure $\mathrm{S4}$ ). In addition to the $\mathrm{Ag} @ \mathrm{CeO}_{2}$ sample, we investigated also a reference sample, which consists of a $10 \mathrm{~nm}$ cerium oxide film without $\mathrm{Ag} \mathrm{NPs}$ on a quartz substrate.

To investigate shape, size distribution and crystal quality of $\mathrm{Ag}$ NPs, ex-situ scanning transmission electron microscopy (STEM) in annular dark field mode and high-resolution transmission electron microscopy (HRTEM) measurements were performed using a JEOL JEM-2200FS microscope (IMEM-CNR, Parma) equipped with a Schottky emitter working at $200 \mathrm{keV}$ (point resolution $0.19 \mathrm{~nm}$ ). All of the images were analyzed using ImageJ software. ${ }^{33}$ The sample used for TEM measurements was grown by co-evaporating $0.05 \mathrm{ML}$ of $\mathrm{Ag} \mathrm{NP}$ and $2 \mathrm{~nm}$ of $\mathrm{CeO}_{2}$ on a carbon-coated copper grid. The nanocluster source conditions were the same used to grow the sample for the optical characterization, except for the lower density of Ag NPS, chosen jn order to distinguish individual $\mathrm{NP}_{\text {g }}$ and to allow for a more detailed characterization.

Steady UV-Vis spectrophotometry measurements were performed using a white non-polarized light source generated by a Xenon lamp equipped with an ORIEL-MS257 monochromator and a silicon photodetector (with a 250-750 $\mathrm{nm}$ range of detection). The samples were oriented so that the sample surface normal formed an incident angle of $22^{\circ}$ with the incident photon beam. We evaluated the absorbance $A$ by measuring the fraction of transmitted $T$ and specular reflected $R$ light $(A=1-T-R)$

The MNPBEM toolbox developed by Hohenester and coworkers, ${ }^{34}$ based on the boundary element method (BEM) approach developed by García de Abajo et al. ${ }^{35}$ was used to calculate the absorption, scattering and extinction coefficients of metal NPs embedded in and sitting on $\mathrm{CeO}_{2}$. The dielectric constants for $\mathrm{CeO}_{2}$ and $\mathrm{Ag}$ were taken from ref. 36 and 37 respectively.

The ultrafast carrier dynamics of our samples were studied by FTAS using a pump-probe femtosecond laser system consisting of a chirped pulse amplifier seeded by a Ti:Sa oscillator $(800 \mathrm{~nm}$, $1 \mathrm{KHz}, 4 \mathrm{~mJ}, 35 \mathrm{fs}) .{ }^{38}$ The pump pulses were generated by an optical parametric amplifier (OPA), pumped by the amplifier The probe for the TA measurements is a white light ha eliminato: also

ha eliminato: of

ha eliminato: of

ha eliminato: deposited

ha eliminato: but a smaller

ha eliminato: $t$

ha eliminato: was

ha eliminato: $i$

ha eliminato: $s$

ha eliminato: obtain

ha eliminato: forms

ha eliminato: Dielectric

ha eliminato: are

ha eliminato: also 

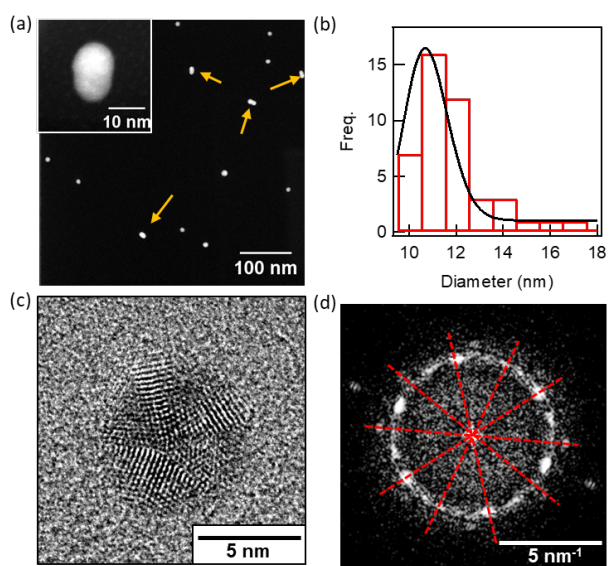
Diameter $(\mathrm{nm})$

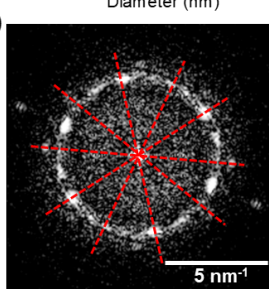

Figure 1. TEM images of $\mathrm{Ag} @ \mathrm{CeO}_{2}:$ a) large scale STEM image, the detail of a NP formed by the coalescence of two single NPs is shown in the inset; b) size distribution evaluated from a series of STEM images and fit by a lognormal curve; c) HRTEM of a single NP; d) Fourier transform of the image in c).

supercontinuum generated in a Fast Transient Spectrometer of IB Photonics (FemtoFrame II). The probe wavelengths ranged between 260 and $760 \mathrm{~nm}$, while the pump-probe delay time was scanned up to $500 \mathrm{ps}$. The probe energy range was covered focusing the fundamental $(800 \mathrm{~nm})$ or the second harmonic $(400 \mathrm{~nm})$ radiation of the amplifier into a rotating $\mathrm{CaF}_{2}$ window that generates the white light supercontinuum in the 320-780 $\mathrm{nm}$ (visible probe) and 260-380 $\mathrm{nm}$ (UV probe) energy ranges, respectively. The IRF was measured to be approximately $50 \mathrm{fs}$. This setup allowed both the energy regions of the LSPR of $A g$ NPs and the cerium oxide band gap to be probed. The study of electron injection efficiency was performed using different pump fluences ranging from 45 to $900 \mu \mathrm{J} / \mathrm{cm}^{2}$.

\section{Results and discussion}

The plasmonic properties of metal NPs are strongly dependent on their size and shape. For this reason, a detailed characterization of the sample morphology is mandatory to understand the origin of the optical absorption features. We therefore investigated an $\mathrm{Ag} @ \mathrm{CeO}_{2}$ sample made of NPs with the same size and a lower density, compared to the one used for the optical studies, to be able to analyze individual NPs by TEM/STEM (see Methods). Figure 1a shows a representative STEM image of a portion of the sample. The NP size distribution shown in Figure $1 p$, was obtained by considering the minimum Feret diameter, evaluated using ImageJ software ${ }_{14}^{33}$ on a series of images. The size distribution has a maximum around $10.5 \mathrm{~nm}$ and a FWHM of $2.4 \mathrm{~nm}$. HRTEM , was used to obtain detailed information on the NPs shape and crystallinity. Most of the individual NPs have a polyhedral shape and a multi-twinned structure_(see Supporting Information for details). As an example we show the HRTEM image of a NP with a five-fold symmetry (Figure 1c), possibly originating from a decahedral shape (see Supporting Information) and the correspondin Fourier transform with a ten-fold symmetry (Figure 1d). As observed in previous works, these phases are thermodynamically metastable and they can be stabilized by fast quenching rates for fcc metal NPs. ${ }^{39,40}$ The image in Figure 1 c shows a well-defined crystal structure only in some portions of the NP, while other areas show a more uniform contrast, which indicates a lower degree of crystalline order, Figure 1 a also shows a non-negligible presence of agglomerates of two or also shows a non-negligible presence of agglomerates of two or
three merged NPs (yellow arrows), one of which is reported in detail in the inset. Some degree of coalescence of NPs grown by the physical method used here has already been reported ${ }^{41}$ and it was ascribed to a non-negligible probability for the NPs to merge either in-flight or on the substrate after the deposition. The granular features evident on the dark area in the inset $d f$ Figure $1 \mathrm{a}$, are ascribed to the presence of a cerium oxide layer covering the substrate and the NPs, as confirmed also by energy dispersive X-ray analysis (data not shown).

Figure 2. Absorbance spectra of quartz (red line), $\mathrm{CeO}_{2}$ film (blule line) and $\mathrm{Ag} @ \mathrm{CeO}_{2}$ sample (green line), shows the static UV-Vis absorbance spectra of the quartz substrate_(red line), , of the $\mathrm{CeO}_{2}$ thin film (blue line) and of the $\mathrm{Ag} @ \mathrm{CeO}_{2}$ sample (green line). Here we define absorbance by the formula $A=1-T-R$ where $T$ is the transmittance and $R$ is the specular reflectance (the $U V$ Vis transmittance and reflectance spectra are reported in the Supporting Information as Figure S2). As expected, the quartz substrate does not show significant absorption in the UV-Vis range, while the $\mathrm{CeO}_{2}$ thin film shows strong absorption dt wavelengths below $350 \mathrm{~nm}$, where the band edge absorption sets in. The extraction of the optical band gap from a Tauc pldt of $(\alpha h v)^{1 / 2} \mathrm{vs}$. hv yields a value of $3.44 \pm 0.03 \mathrm{eV}$ (see Supporting Information, Figure S3) that is in good agreement with the range of values $3.0-3.6 \mathrm{eV}$ obtained for ceria films prepared by sputter deposition or thermal evaporation..$^{42,43}$ In general, a red shift of the optical band gap from the pure bulk value is indicative of the presence of $\mathrm{Ce}^{3+}$ and oxygen defects.44-46 Nonethetess, the $\mathrm{CeO}_{2}$ fitm used here is of good quality, as wheh defects are intentionally added much larger optical shifts are observed. ${ }^{47}$ The analysis of the Ce $3 \mathrm{~d}$ XPS lineshape (see Supporting Information, Figure S4) demonstrates a stoichiometry close to $\mathrm{CeO}_{2}$ for the ceria layer. The presence of a small absorbance in the visible region (approx. $5 \%$ ) in the $\mathrm{CeO}_{2}$ film spectrum can be assigned to the roughness of the thin film, which gives rise to some diffuse reflectance and consequently to a non-negligible apparent absorbance. The

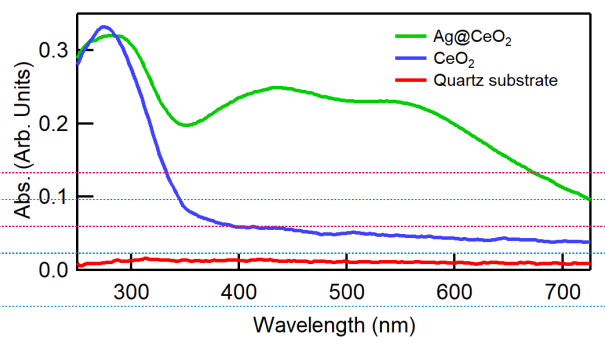

Figure 2. Absorbance spectra of quartz (red line), $\mathrm{CeO}_{2}$ film (blue line) and $\mathrm{Ag} @ \mathrm{CeO}_{2}$ sample (green line). ha formattato: Tipo di carattere: Non Grassetto, Proporzioni car $108 \%$

ha eliminato: Figure 1 Figure 1

ha formattato: Tipo di carattere: Non Grassetto, Proporzioni car $108 \%$

ha eliminato: (see Figure S1, Supporting Information)

ha formattato: Tipo di carattere: Non Grassetto, Proporzioni car $108 \%$

ha eliminato: Figure 1Figure 1

ha formattato: Tipo di carattere: Non Grassetto, Proporzioni car $108 \%$

ha eliminato: Figure $1 \mathrm{~d}$

ha eliminato: figure

ha eliminato: $d$

ha formattato: Tipo di carattere: Non Grassetto, Proporzioni car $108 \%$

ha formattato: Tipo di carattere: Non Grassetto, Proporzioni car $108 \%$

ha eliminato: Figure 2. Figure 2

ha formattato: Tipo di carattere: Non Grassetto, Proporzioni car $108 \%$

ha formattato: Tipo di carattere: Non Grassetto

ha eliminato: ;

ha eliminato: detail of a NP formed by the coalescence of two single NPs.

ha eliminato:

ha eliminato: a

ha eliminato: a

ha eliminato: $E$

ha eliminato: of

ha formattato: Tipo di carattere: Non Grassetto, Proporzioni car $108 \%$

ha eliminato: Figure 1Figure 1

ha formattato: Tipo di carattere: Non Grassetto, Proporzioni car $108 \%$

ha eliminato: , used to evaluate $t$

ha formattato: Tipo di carattere: Non Grassetto, Proporzioni car $108 \%$

ha eliminato: Figure 1 Figure 1

ha formattato: Tipo di carattere: Non Grassetto, Proporzioni car $108 \%$

ha formattato: Apice

ha formattato: Non Apice / Pedice

ha eliminato: (Figure 1c) 


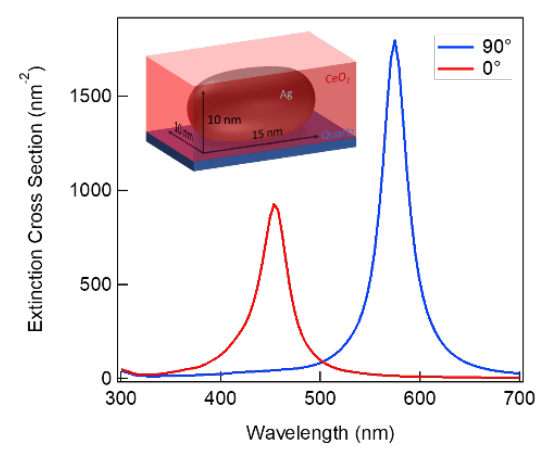

Figure 4 , Simulated extinction cross section spectra obtained using the MNPBEM toolbox ${ }^{34}$ with a linearly polarized light parallel (red curves) and perpendicular (blue curves) to the minor axis of embedded ellipsoidal NPs with geometry $15 \times 10 \times 10 \mathrm{~nm}^{3}$ embedded in a $10 \mathrm{~nm}$ thick ceria film.

incorporation of $\mathrm{Ag}$ NPs into the $\mathrm{CeO}_{2}$ film significantly modifies the absorbance spectrum with the appearance of two broad peaks with maxima at 420 and $580 \mathrm{~nm}$, assigned to the LSPR in Ag NPs, shifted into the visible range due to the high dielectric constant of $\mathrm{CeO}_{2}$. The presence of two peaks can be either due to an anisotropic NPs shape, giving rise to transverse and longitudinal LSPRs at different wavelengths, or to the superposition of LSPRs and delocalized plasmon resonances involving closely spaced interacting spherical NPs. We have ruled out the second possibility because the average distance between the Ag NPs in our samples, estimated using the measured deposited amount of NPs (see Methods) and their average size, is too large to induce an extended plasmon resonance (see Supporting Information, Figure S5).48,49 The STEM images in Figure 1 show the presence of a fraction of anisotropically shaped NPs formed by the coalescence of individual Ag NPs during the deposition process. We tentatively ascribe the presence of two peaks in the $\mathrm{Ag} @ \mathrm{CeO}_{2}$ absorbance spectra to the NP anisotropy.

The MNPBEM toolbox developed by Hohenester and coworkers, ${ }^{34}$ was used to confirm the origin of the two LSPR peaks by simulating the absorption spectrum of the $\mathrm{Ag} @ \mathrm{CeO}_{2}$ sample. The shape of the NPs observed by TEM/STEM was approximated using different geometries, including nanorods, spheroids, ellipsoids and nanodisks (see Supporting Information, Figure S5). The best agreement with the experimental data was obtained using ellipsoidal NPs with 10 and $15 \mathrm{~nm}$ in-plane axes and $10 \mathrm{~nm}$ out-of-plane axis, embedded within a $10 \mathrm{~nm}$ thick $\mathrm{CeO}_{2}$ film $\mathrm{m}_{*}$ This geometry is compatible with the measured average size and shape of the NPs and with the thickness of the oxide matrix of the sample jnvestigated here. The calculated extinction cross section spectra for this configuration, reported in Figure 3, show two peaks at $450 \mathrm{~nm}$ and $590 \mathrm{~nm}$ with the light polarization respectively parallel and perpendicular to the minor axis of the ellipsoid. Based on the results of the simulations, the two broad peaks with maxima at 450 and $580 \mathrm{~nm}$ in the experimental spectra acquired using non-polarized light can be assigned to the excitation of the two LSPRs in the anisotropic Ag NPs embedded within the cerium oxide matrix. The large width and different relative intensity of the peaks observed in the experimental spectra are ascribed to the aspect ratio distribution of the NPs. Figure $4 \mathrm{~b}$ shows the false-color map of distribution of the NPs. Figure $4 \mathrm{~b}$ shows the false-color map of transient absorbance (TA) spectra of the $\mathrm{CeO}_{2}$ film without $\mathrm{Ag}$
NPs, excited with a pump at $275 \mathrm{~nm}(4.5 \mathrm{eV})$, significantly above the optical band gap of the $\mathrm{CeO}_{2}$ film. The map has been constructed by combining the maps recorded using the UV $(285-350 \mathrm{~nm})$ and visible $(340-750 \mathrm{~nm})$ white light supercontinuum probes. It reports the TA intensity as a function of the probe wavelength ( $y$-axis) and of the time delay between pump and probe ( $x$-axis), and it provides information about the dynamics of the photoexcited states in the $\mathrm{CeO}_{2}$ sample. The TA map is dominated by two main features: a negative peak at 300 $\mathrm{nm}$ and a positive peak around $350 \mathrm{~nm}$. The two features show only a slight decrease in intensity with increasing delay time up to $500 \mathrm{ps}$, which suggests that the corresponding excited electrons are stable to recombination, possibly due to trapping in localized states. ${ }^{50}$ The negative peak corresponds to the wavelength of the maximum of the absorbance spectrum of $\mathrm{CeO}_{2}$ (Figure 2) and it is ascribed to photoinduced bleaching (PB) of the $\mathrm{O} 2 \mathrm{p}$ band. Considering the band structure of $\mathrm{CeO}_{2}$ with a valence band of $\mathrm{O} 2 \mathrm{p}$ character, a conduction band of $\mathrm{Ce} 5 \mathrm{~d}$ character and Ce $4 \mathrm{f} \mathrm{levels} \mathrm{in} \mathrm{between} \mathrm{(Figure} \mathrm{4a),} \mathrm{we} \mathrm{assign} \mathrm{the}$ positive peak at $345 \mathrm{~nm}(3.3 \mathrm{eV})$ to photoinduced absorption of electrons excited by the pump from the valence band to ce $4 \mathrm{f}$ levels and further excited into the $\mathrm{Ce} 5 \mathrm{~d}$ band by the probe. This assignment is supported also by the smaller energy separation between the $4 \mathrm{f}$ and $5 \mathrm{~d}$ bands $\left(\mathrm{E}_{4 \mathrm{f}-5 \mathrm{~d}} \sim 3 \mathrm{eV}\right)$ in stoichiometric $\mathrm{CeO}_{2},{ }^{51,52}$ compared to the energy separation between filled $\mathrm{O}$ $2 p$ and empty $\mathrm{Ce} 4 \mathrm{f}\left(\mathrm{E}_{2 \mathrm{p}-4 \mathrm{f}} \sim 3.3 \mathrm{eV}\right.$ 51,52) states, compatible with the higher energy of the ground state bleaching compared to

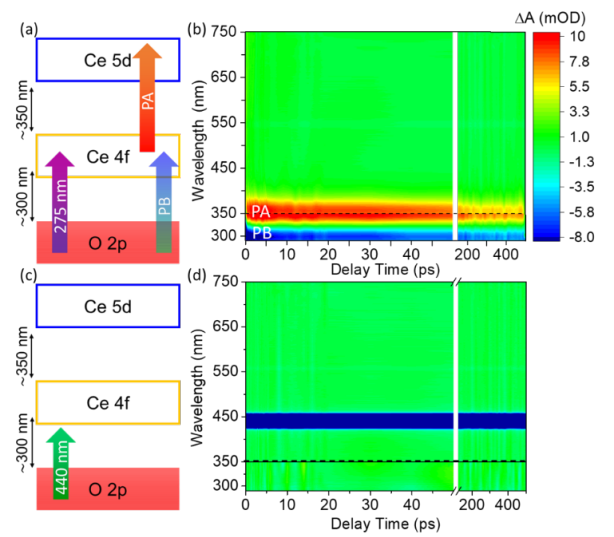

Figure $3_{-}$, Sketch of the cerium oxide electronic structure ${ }_{L}$ showing (a) the transitions induced by the pump at $275 \mathrm{~nm}$ and the corresponding TA signals and $(\mathrm{f})$ the absence of transitions with the pump at $440 \mathrm{~nm}$. False-color map of the TA spectra for the $\mathrm{CeO}$ sample excited with a pump at $275 \mathrm{~nm}$ (b) and at $440 \mathrm{~nm}$ (d) and probed with both UV and visible white light supercontinuum probes at different delay times (from 3 to $500 \mathrm{ps}$ ); the black dashed line at $350 \mathrm{~nm}$ separates the two sets of data acquired with the two different probes. The blue intense line centered at $440 \mathrm{~nm}$ is the scattered light from the pump. ha eliminato: is

ha eliminato: Figure 4 Figure 4

ha eliminato:

ha eliminato: 3

ha eliminato: Figure $4 \mathrm{~b}$

ha eliminato: Figure 4 Figure 4

ha eliminato: absorption

\section{ha eliminato: ,}

ha eliminato: well

ha eliminato: 4

ha eliminato: a) False-color map of the TA spectra for the $\mathrm{CeO}_{2}$ sample excited with a pump at $275 \mathrm{~nm}$ and probed with both UV and visible white light supercontinuum probes at different delay times (from 3 to $500 \mathrm{ps}$ ): the black dashed line at $350 \mathrm{~nm}$ separates the two sets of data acquired with the two different probes. b)

\section{ha eliminato: here}

ha eliminato: $b$

ha eliminato: 

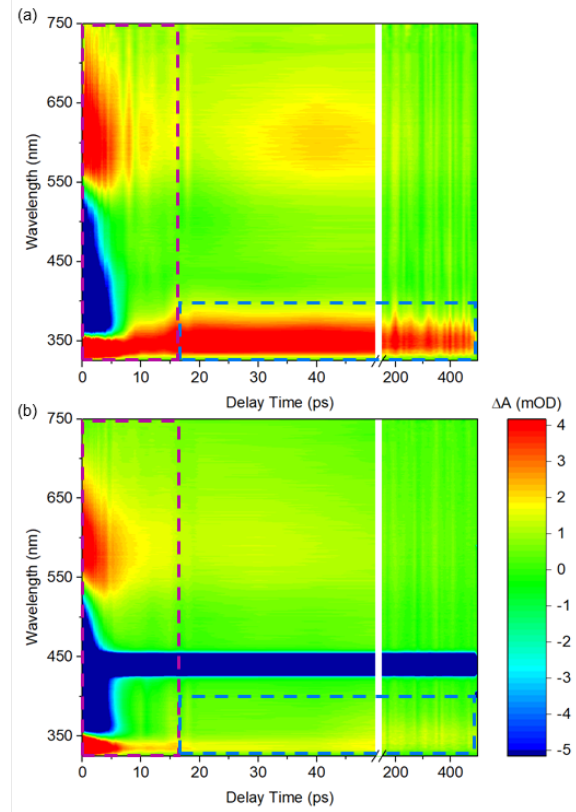

Figure 5. False-color maps of the TA spectra obtained for the $\mathrm{Ag} @ \mathrm{CeO}_{2}$ sample excited with a pump (a) at $275 \mathrm{~nm}$, i.e. above the $\mathrm{CeO}_{2}$ band gap, and (b) at $440 \mathrm{~nm}$ i.e. below the $\mathrm{CeO}_{2}$ band gap at LSPR maximum. The red box highlights the part of the map dominated by the plasmonic response of the Ag NPs while the blue box contains the signals assigned to electron injection into the $\mathrm{CeO}_{2}$ film. The blue intense line centered at $440 \mathrm{~nm}$ is the scattered ligh from the pump.

the photoinduced absorption features in the TA spectra reported in Figure $4 \mathrm{~b}$. Indeed, the density of states in the valence band slightly depends on the average oxidation state of cerium ions and on the density and kind of defects. Figure $4 \mathrm{~d}$ reports the TA map acquired on the same $\mathrm{CeO}_{2}$ sample, using a pump below the band gap threshold $(440 \mathrm{~nm})$. The absence of TA features is in agreement with the assignment made for the features in the TA map in Figure 4a. The negative intensity in the 425-455 $\mathrm{nm}$ wavelength range is due to scattering of th photons with a $440 \pm 15 \mathrm{~nm}$ wavelength used for the pump. Figure 5 a shows the false-color map of the TA spectra obtained for the $\mathrm{Ag} @ \mathrm{CeO}_{2}$ sample using a pump at $275 \mathrm{~nm}$, above the band gap of $\mathrm{CeO}_{2}$, and the visible probe. The map shows new TA features at delay times shorter than 10 ps (Figure 5a): a broad positive band around $610 \mathrm{~nm}$, a broad negative band at $380-520 \mathrm{~nm}$ and a sharp positive band below $350 \mathrm{~nm}$ (see also Supporting Information, Figure S7). These features are assigned to the LSPR of Ag NPs, and they present the typical decay times of transient plasmonic signals ( $<10-15 \mathrm{ps}$ ). The signals originate from the heating induced by interband excited electrons, which promote strong changes in the dielectric constant of the material and thus a change of the LSPR response of the NPs 53 . This change typically leads to a bleaching of the central part of the resonance and to a simultaneous increased TA intensity in the wings. ${ }^{48}$ At delay times larger than 10 ps the map in Figure 5 a presents only the positive photoinduced absorption peak assigned to $\mathrm{CeO}_{2}$ and already discussed for the bare $\mathrm{CeO}_{2}$ film (Figure $4 \mathrm{~b}$ ), which persists in all the delay time range investigated, i.e. up to $500 \mathrm{ps}$. Figure $5 \mathrm{~b}$ reports the false-coldr map of the TA spectra obtained for the $\mathrm{Ag} @ \mathrm{CeO}_{2}$ sample with a pump at $440 \mathrm{~nm}$, below the band gap of $\mathrm{CeO}_{2}$, and the visible probe. The pump energy corresponds to LSPR excitation in the Ag NPs (Figure 2). Also in this case at delay times shorter than Ag NPs (Figure 2). Also in this case at delay times shorter than
10 ps LSPR signals are observed, similar to the ones observed in the TA map obtained with the pump at $275 \mathrm{~nm}$ (Figure 5a). The main point to note is that the map of the $\mathrm{Ag} @ \mathrm{CeO}_{2}$ at time delays higher than $10 \mathrm{ps}$ (Errore. L'argomento parametro sconosciuto b) exhibits the same persistent signal at $345 \mathrm{~nm}$ assigned to photoinduced absorption in $\mathrm{CeO}_{2}$, although the pump energy is below the $\mathrm{CeO}_{2}$ band gap. The presence of the photoinduced absorption signal is ascribed to transient occupation of $4 \mathrm{f}$ levels in $\mathrm{CeO}_{2}$ induced by LSPR-mediated electron injection. Figure $4 \mathrm{~d}$ clearly shows that in the Ced sample without $\mathrm{Ag}$ nanoparticles the intensity at $345 \mathrm{~nm}$ is zero with a pump at $440 \mathrm{~nm}$, supporting the hypothesis made on the origin of the TA signal.

To obtain further information on the electron injection mechanism and on its efficiency, we compared the time evolution of the photoinduced absorption signal in the different samples, excited at the different pump energies, by considering horizontal cuts of the TA maps at $345 \mathrm{~nm}$ (Figure 6). The TA of the $\mathrm{CeO}_{2}$ film pumped above its band gap (Figure 6a) increases very rapidly within the first $5 \mathrm{ps}$ and it slowly decays at longer times. The signal was fitted using a linear combination of a rising decreasing exponential function to obtain the slow decay. Each function was fonvolved with the instrument response function (see Supporting Information for details). The fitting function obtained js used as a fingerprint of ceria de-excitation in the fits of the TA decay of the other samples. The photoinduced absorption signal for the $\mathrm{Ag} @ \mathrm{CeO}_{2}$ sample pumped at $275 \mathrm{~nm}$ (Figure $6 \mathrm{~b}$ ) shows an additional LSPR-related decrease of intensity below 10 ps (the LSPR positive wing), which is described by introducing into the fit a further exponentially decreasing function with a short decay time. The signal of the $\mathrm{Ag} @ \mathrm{CeO}_{2}$ sample pumped at $440 \mathrm{~nm}$ (Figure 6c) was fitted by a linear combination of the ceria-related and LSPR-relate functions, obtaining very similar decay times (see table S1, Supporting Information). The good quality of the fit (green line in Figure $\mathrm{G}_{\mathrm{c}}$ ) is consistent with the transient occupation of the Ce $4 \mathrm{f}$ levels when the $\mathrm{Ag} @ \mathrm{CeO}_{2}$ system is pumped below its bandgap at the Ag LSPR wavetength, and it indicates a plasmonmediated charge transfer between the Ag NPs and the oxide. The dynamics of the electron injection at delay times shorter than 5 ps cannot be accessed, because the $\mathrm{CeO}_{2}$-related signal is superimposed with the TA-signal related to the LSPR of the Ag NPs (see also Supporting Information, Figure -57 )

A quantification of the injection efficiency was obtained by comparing the TA signals at $345 \mathrm{~nm}$ of the $\mathrm{Ag} @ \mathrm{CeO}_{2}$ sample pumped at different energies below the band gap, with the same signal on $\mathrm{CeO}_{2}$ film pumped at $275 \mathrm{~nm}$, in the $50-250 \mathrm{ps}$ delay time range, i.e. after the decay of the LSPR-related transient signals. In this delay time range, the photoinduced absorption signal of $\mathrm{CeO}_{2}$ dominates and its intensity is proportional to the density of electrons in the cerium oxide $4 \mathrm{f}$

\section{ha formattato: Pedice}

ha formattato: Pedice

ha eliminato: Figure 4Figure 4

ha formattato: Tipo di carattere: Non Grassetto, Inglese americano, Proporzioni car $108 \%$

ha formattato: Tipo di carattere: Non Grassetto, Inglese americano, Proporzioni car $108 \%$

ha eliminato: Figure 5 Figure 5

ha formattato: Tipo di carattere: Non Grassetto, Inglese americano, Proporzioni car $108 \%$

ha formattato: Tipo di carattere: Non Grassetto, Inglese americano, Proporzioni car 108\%

\section{ha formattato: Evidenziato}

ha eliminato: Error! Unknown switch argument.

ha eliminato: above (

ha eliminato: )

ha eliminato: convoluted

ha eliminato: below (

ha eliminato: )

ha eliminato: fitting function

ha eliminato:

ha formattato: Tipo di carattere: Non Grassetto, Proporzioni car $108 \%$

ha eliminato: Figure 6 Figure 6

ha formattato: Tipo di carattere: Non Grassetto, Proporzioni car $108 \%$

ha eliminato: the use of

ha eliminato: Figure 5 Figure 5

ha formattato: Tipo di carattere: Non Grassetto, Inglese americano, Proporzioni car 108\%

ha formattato: Tipo di carattere: Non Grassetto, Inglese americano, Proporzioni car $108 \%$

ha formattato: Pedice

ha formattato: Pedice

ha formattato: Tipo di carattere: Non Grassetto, Inglese americano, Proporzioni car $108 \%$

ha eliminato: Figure 5 Figure 5

ha formattato: Tipo di carattere: Non Grassetto, Inglese americano, Proporzioni car 108\% 
band after the excitation. Assuming that each photon with energy higher than the bandgap excites an electron from the valence band to the $\mathrm{Ce} 4 \mathrm{f}$ levels, to obtain the injection efficiency we compared the number of excited electrons per
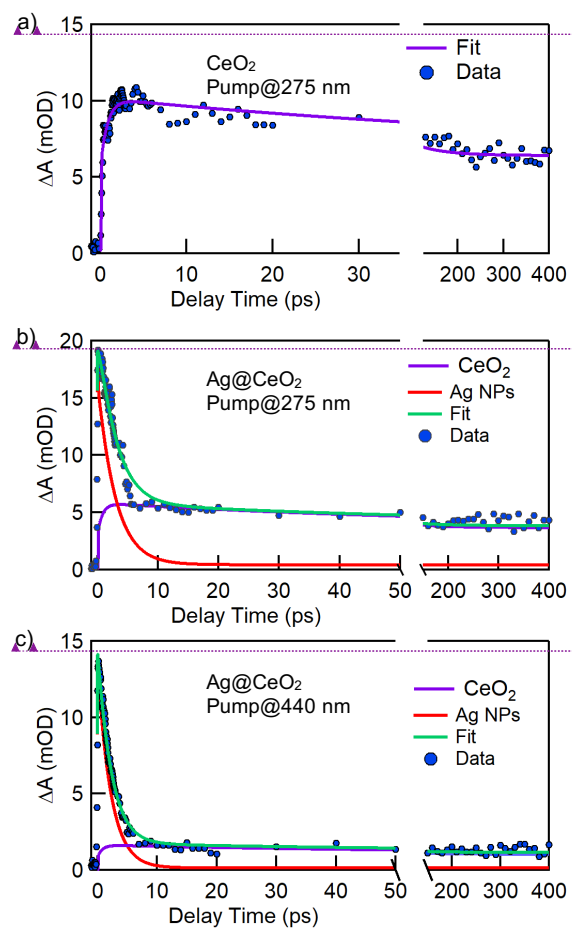

Figure 6. Time dependence of transient excited state signal at 345 $\mathrm{nm}$ of the $\mathrm{CeO}_{2}$ sample pumped at $275 \mathrm{~nm}$ (a) and of the $\mathrm{Ag} @ \mathrm{CeO}_{2}$ sample pumped at $275 \mathrm{~nm}$ (b) and at $440 \mathrm{~nm}$ (c). The components used for data fitting, related to photoinduced absorption in $\mathrm{CeO}_{2}$ (purple line) to plasmonic deexcitation (red line), and the full fitting spectrum (green line) are also shown as solid lines.

incident photon in the $\mathrm{Ag} @ \mathrm{CeO}_{2}$ sample at different pump energies below the band gap to the number obtained for the $\mathrm{CeO}_{2}$ film with a pump above the band gap.

We estimated the incident photon density at the different pump fluences and energies with the formula:

$$
\boldsymbol{n}_{\text {ph }}=\frac{\boldsymbol{A}\left(\lambda_{\text {pump }}\right) \cdot \boldsymbol{F}}{\boldsymbol{D} \cdot \hbar \omega_{\text {pump }}},
$$

where $\boldsymbol{A}\left(\lambda_{\text {pump }}\right)$ is the absorbance of the sample at the pump energy, $F$ is the pump fluence, $D$ is the total sample thickness and $\hbar \omega_{\text {pump }}$ is the pump energy. ${ }^{9}$ Then, we correlate this result with the density of electrons in the $4 \mathrm{f}$ levels, obtained by calculating the integral of the photoinduced absorption signal in the 50-250 ps time interval. The results for the $\mathrm{CeO}_{2}$ film pumped at $275 \mathrm{~nm}$ and for the $\mathrm{Ag} @ \mathrm{CeO}_{2}$ sample pumped at 440 $\mathrm{nm}$ are shown in Figure 7 a together with their linear fits, obtained by fixing the intercept to 0 mOD.ps (see Supporting Information for further details). The slopes $k$ of the lines as in Figure $7 \mathrm{a}$ are proportional to the electron population in the 4 levels at each pump energy. The ratio between the $\kappa$ values for the $\mathrm{Ag} @ \mathrm{CeO}_{2}$ sample and the reference $\mathrm{CeO}_{2}$ film gives the efficiency of the charge injection from Ag-NP.s- to ceritum oxide. The resulting injection efficiencies at the different pump energies are shown in Figure $7 \mathrm{~b}$. Surprisingly high values in the $12-16 \%$ range have been obtained for excitation between 400 and $500 \mathrm{~nm}$, while a lower value of $6 \%$ is found for the pump at $600 \mathrm{~nm}$.

To understand such high efficiencies we first consider mechanisms such as light trapping ${ }^{18}$ energy transfer to the semiconductor through near-field enhancement, 19 plasmon induced radiative energy transfer, ${ }^{19}$ and plasmon induced resonant energy transfer. ${ }^{19}$ The similar slopes of the $\mathrm{Ag} @ \mathrm{CeO}_{2}$ sample and $\mathrm{CeO}_{2}$ film pumped at $275 \mathrm{~nm}$ reported in Figure 7 a indicate a similar excitation of the ceria with and without A negligible contribution to the injection efficiency, as expected for such small NPs. ${ }^{18}$ All the mechanisms considered up to now require an overlap between the LSPR and an optical transition in the semiconductor and they were not expected to contribute significantly in the sample here considered.
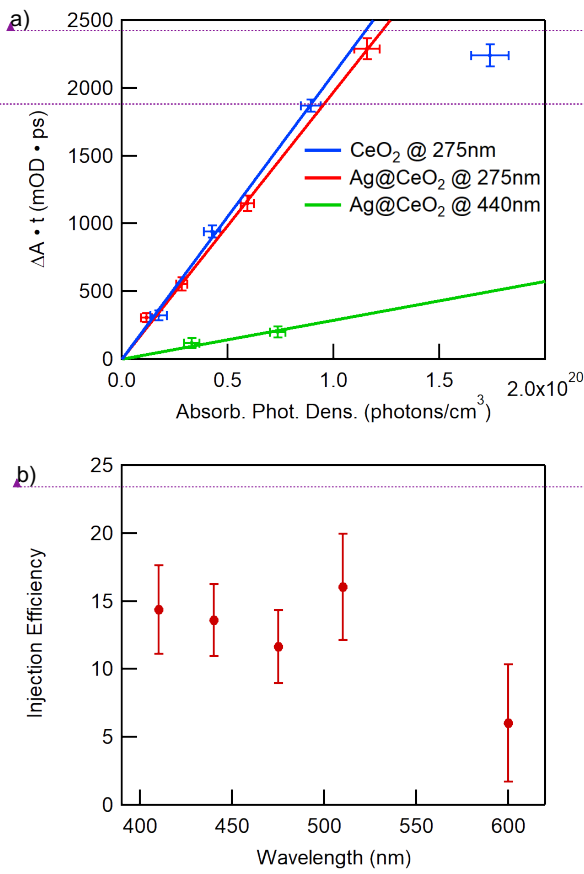

Figure 7. a) $\Delta A \cdot t$ vs absorbed photon density and b) injection efficiency as a function of pump wavelength. ha formattato: Tipo di carattere: Non Grassetto, Proporzioni car $108 \%$

ha eliminato: Figure 7Figure 7

ha formattato: Tipo di carattere: Non Grassetto, Proporzioni car $108 \%$

ha formattato: Tipo di carattere: $9 \mathrm{pt}$

ha formattato: Tipo di carattere: $8 \mathrm{pt}$

ha formattato: Tipo di carattere: Non Grassetto, Proporzioni car $108 \%$

ha eliminato: Figure 7Figure 7

ha formattato: Tipo di carattere: Non Grassetto, Proporzioni car $108 \%$

ha eliminato: Figure 7Figure 7

ha formattato: Tipo di carattere: Non Grassetto, Proporzioni car $108 \%$

ha formattato: Tipo di carattere: Non Grassetto, Proporzioni car $108 \%$

ha formattato: Tipo di carattere: $9 \mathrm{pt}$

ha formattato: Tipo di carattere: $8 \mathrm{pt}$

ha formattato: Tipo di carattere: 9 pt

ha formattato: Tipo di carattere: $9 \mathrm{pt}$

ha formattato: Tipo di carattere: $8 \mathrm{pt}$ 
We therefore concentrate our discussion on the mechanisms which do not require an overlap between the LSPR of the metal NPs and an optical transition in the semiconductor, such as plasmon-induced indirect hot electron injection ${ }^{2}$ and plasmoninduced direct electron injection. ${ }^{22,54}$ Until recently it was thought that only the first of these mechanisms could induce an electron injection in the oxide. The dephasing and the decay of the LSPR excitation inside the NPs lead to a non-thermal energy distribution of hot electrons, followed by injection of a part of the distribution over/through the Schottky barrier formed at the interface between the metal and the semiconductor due to band bending. A large body of research in the literature is dedicated to this mechanism, spawning an entire field of research called hot carrier science. ${ }^{20,55,56}$ Indeed, numerous systems have been identified in which this mechanism takes place. The efficiency of this process is intrinsically limited by competition with electron thermalization within the metal leading to a hot Fermi-Dirac energy distribution in which most electrons do not have sufficient energy to overcome the Schottky barrier. Furthermore, the electron initially formed must have its moment vector directed towards the interface in order to be injected into the semiconductor. White and Catchpole estimated a maximum injection efficiency of $8 \%$ fo this indirect plasmonic mechanism in the case of Au@ $\mathrm{TiO}_{2} .{ }^{57} \mathrm{It}$ should be noted that $\mathrm{Ag}$ is known to be more efficient at generating hot electrons than Au 58 Nonetheless the true when considering the optimistic assumptions, such as a flat energy distribution of non-thermal electrons formed by LSPR decay up to the energy of the absorbed photon, employed in the above estimate. Based on these considerations, we suggest that other processes in addition to hot electron injection are at work in this system.

The plasmon-induced direct electron injection mechanism, which was theoretically proposed by Long and Prezhdo ${ }^{54}$ to have a very high efficiency of $50 \%$ in the $\mathrm{Au}_{0} \mathrm{TiO}_{2}$ system, is the instantaneous generation of charge-separated states across the metal/semiconductor interface following the dephasing of the LSPR. This prediction was confirmed by the measurement of an electron injection efficiency of $24 \%$ in the Au@CdSe system. ${ }^{22}$ Furthermore, Tan et al. ${ }^{59}$ experimentally demonstrated in the $\mathrm{Au} @ \mathrm{TiO}_{2}$ system that the direct dephasing of the LSPR excitation in Au formed electrons in $\mathrm{TiO}_{2}$ on the sub-10 fs timescale. ${ }^{23}$ It is possible that the high effici injection observed in the $\mathrm{Ag} @ \mathrm{CeO}_{2}$ system are partly due to this direct mechanism.

A further point to be discussed is how the injection efficiency varies with pump wavelength. We observe a drop in the injection efficiency at $600 \mathrm{~nm}$ with respect to that in the 400 $500 \mathrm{~nm}$ range (see Figure $7 \mathrm{~b}$ ), even though the absorption cross section remains quite constant (see Figure 2 ). In the case of the indirect injection mechanism one could justify this observation if the LSPR excited at $600 \mathrm{~nm}$ produces fewer hot electrons with energy sufficient to overcome the barrier between the metal and the semiconductor than excitation in the $400-500 \mathrm{~nm}$ range. Based on photon energy considerations alone this is certainly possible, however it has also been observed that the efficiency of hot electron generation depends on the intensity of the hot spots of the NPs. In the system studied here, the hot spots generated for excitation of the longitudinal LSPR resonance at $580 \mathrm{~nm}$ are significantly more intense than those generated at the transverse resonance at $420 \mathrm{~nm}$. This is
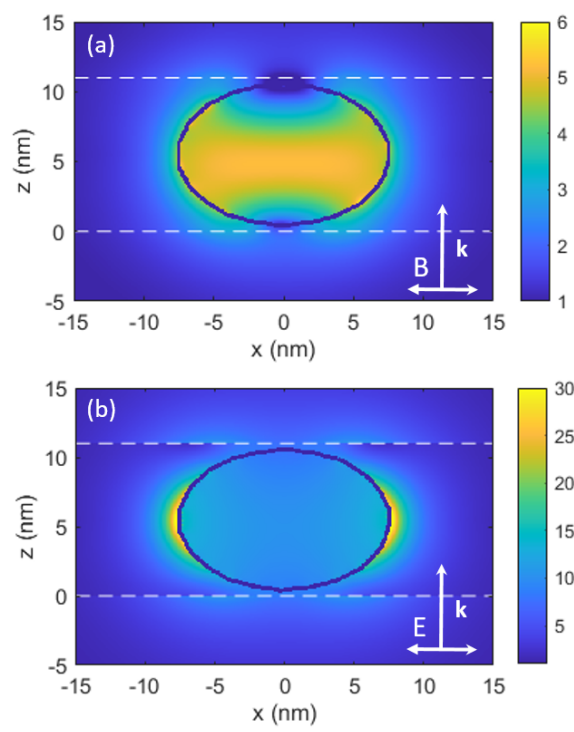

Figure 8. Field enhancement, defined as $\left|E / E_{0}\right|$ (where the $E_{0}$ is the electric field of the incident radiation and $E$ the resultant one), in ellipsoidal Ag NP with $15 \mathrm{~nm}$ major axis and $10 \mathrm{~nm}$ minor axes, embedded in $11 \mathrm{~nm}$ of ceria, in the case of incident radiation (a) with electric field parallel to the minor axis and energy at the LSPR along that direction $(460 \mathrm{~nm})$; (b) with electric field parallel to the major axis and energy at the LSPR along that direction $(590 \mathrm{~nm})$. The filed enhancement in the two cases is so large to prevent the possibility of using a common false color scale.

evident in Figure 8, where the electric near field enhancement simulations (performed using MNPBEM toolbox ${ }^{34}$ ) of the two excitation modes are compared.

The direct injection mechanism is strongly modulated by the coupling of the density of the states in the semiconductor (the if levels with a narrow energy width) and the plasmonic resonances at the interface. For this reason, the resulting injection efficiency does not follow the absorption cross section of the Ag NPs. Depending on the positioning of the Fermi level of the metal within the band gap of the $\mathrm{CeO}_{2}$, some plasmonic excitations at specific energies can be more efficient than others in injecting electrons into the Ce $4 \mathrm{f}$ states. However, it is difficult to make a quantitative and precise estimate of these energies due to the sensitivity of the Fermi level to the crystal structure of the NPs and the uncertainty of the position of the enery of the semiconductor due to interface The observed plasmon-mediated electron injection efficiency is indeed expected to largely influence the properties of the cerium oxide surrounding the Ag NPs. The long-living charges injected in $\mathrm{Ce} 4 \mathrm{f}$ levels will temporarily modify the oxidation state of the corresponding Ce ions from $4+$ to $3+$, decreasing the state neighborhoods. If the oxide layer embedding the Ag NPs is thin enough, surface oxygen vacancies will be easily formed, thus 
increasing the catalytic activity of the material. This mechanism is expected to induce a sensitization of the material to visible light to promote and catalyze redox reactions.

\section{Conclusions}

We have investigated the static and transient optical propertie of Ag NPs embedded within a cerium oxide matrix. The interpretation of the results is based on a detailed morphological characterization. The NPs have an icosahedral shape, a narrow size distribution centered around $10 \mathrm{~nm}$, and they are partially aggregated into clusters of two or more NPs. The system shows a strong absorption band in the visible region, assigned to LSPRs, in agreement with simulations made using a boundary element method approach. Femtosecond using a boundary element method approach. Femtosecond transient occupation of the Ce $4 \mathrm{f}$ levels also at pump energies below the band gap of the cerium oxide. The observed phenomenon is ascribed to plasmon-mediated electron injection from Ag NPs to cerium oxide. The electron injection efficiency was quantified to be in the $6-16 \%$ range and to vary with the pump energy. The high efficiency revealed suggests that the indirect hot electron injection is not the only mechanism active in this system. We therefore suggest that a direct process, in which electrons are injected into the semiconductor by direct dephasing of the plasmonic resonances at the interface, has to be taken into account. The plasmon-mediated charge injection discussed in this work is of great importance for a knowledge-driven design of new efficient ceria-based nanostructured photocatalytic materials.

\section{Conflicts of interest}

There are no conflicts to declare.

\section{Acknowledgements}

DC, PO'K, SDA and SV acknowledge funding from PRIN project no. 2015CL3APH.

SV and SDA acknowledge funding from Università degli Studi di

Modena e Reggio Emilia through FAR2016 project titled:

"Innovative (oxide-based) materials and methods for fuel cell electrodes implementation."

\section{Author Contributions}

PL, POK, DC, SDA and SV conceived the experiment. JSPC, MCS, and SDA prepared the samples and did the XPS analysis. MCS, GB and JSPC did the TEM analysis. JSPC, SB and ADB did the UVVis spectrophotometry analysis. JSPC, DC, POK, LDM, AP did the FTAS experiment. POK and JSPC did the BEM simulations. All authors contributed to data interpretation and to manuscript writing.

\section{References}

2

5

6
7

8

9

10

.

10, 310-315.
N. V. Skorodumova, S. I. Simak, B. I. Lundqvist, I. A. Abrikosov and B. Johansson, Phys. Rev. Lett., 2002, 89, 166601

17 A. Laachir, V. Perrichon, A. Badri, J. Lamotte, E. Catherine, J. C. Lavalley, J. Elfallah, L. Hilaire, F. Lenormand, E. Quemere, G. N. Sauvion and O. Touret, J. Chem. Soc. Trans., 1991, 87, 1601-1609.

18 H. A. Atwater and A. Polman, Nat. Mater., 2010, 9, 205213.

19 X.-C. Ma, Y. Dai, L. Yu and B.-B. Huang, Light Sci. \&Amp; Appl., 2016, 5, e16017.

20 P. Christopher and M. Moskovits, Annu. Rev. Phys. Chem., 2017, 68, 379-398.

21 N. Wu, Nanoscale, 2018, 10, 2679,-2696.

22 K. Wu, J. Chen, J. R. McBride and T. Lian, Science, 2015, 349, 632-635.

23 S. Tan, A. Argondizzo, J. Ren, L. Liu, J. Zhao and H. Petek, Nat. Photonics, 2017, 11, 806-812.

24 S. K. Cushing, J. Li, F. Meng, T. R. Senty, S. Suri, M. Zhi, M. Li, A. D. Bristow and N. Wu, J. Am. Chem. Soc., 2012, 134, 15033-15041.

25 X. Kong, Z. Wang and A. O. Govorov, Adv. Opt. Mater., 2017, 5, 1600594.

26 H. Harutyunyan, A. B. F. Martinson, D. Rosenmann, L. K. Khorashad, L. V Besteiro, A. O. Govorov and G. P. Wiederrecht, Nat. Nanotechnol., 2015, 10, 770-774. ha eliminato:

ha formattato: Tipo di carattere: Grassetto

ha eliminato: steady

ha eliminato: dynamic

ha eliminato: /1-166601/4

ha formattato: Tipo di carattere: Grassetto, Inglese americano

ha formattato: Inglese americano

ha formattato: Inglese americano

ha eliminato: $(80-$.$) .$ 
27 L. V Besteiro and A. O. Govorov, J. Phys. Chem. C, 2016, 120, 19329-19339.

28 J. W. Hong, D. H. Wi, S.-U. Lee and S. W. Han, J. Am. Chem Soc., 2016, 138, 15766-15773.

29 E. Cortés, W. Xie, J. Cambiasso, A. S. Jermyn, R. Sundararaman, P. Narang, S. Schlücker and S. A. Maier, Nat. Commun., 2017, 8, 14880

30 M. W. Knight, Y. Wang, A. S. Urban, A. Sobhani, B. Y. Zheng, P. Nordlander and N. J. Halas, Nano Lett., 2013, 13, 16871692.

31 C. Ng, P. Zeng, J. A. Lloyd, D. Chakraborty, A. Roberts, T. A. Smith, U. Bach, J. E. Sader, T. J. Davis and D. E. Gómez, arXiv Prepr. arXiv1711.07095.

32 P. Luches, F. Pagliuca, S. Valeri, F. Illas, G. Preda and G. Pacchioni, J. Phys. Chem. C, 2012, 116, 1122-1132.

33 C. A. Schneider, W. S. Rasband and K. W. Eliceiri, Nat. Methods, 2012, 9, 671-675.

34 U. Hohenester and A. Trügler, Comput. Phys. Commun., 2012, 183, 370-381.

35 F. J. García de Abajo and A. Howie, Phys. Rev. B, 2002, 65 115418.

36 F.-C. Chiu and C.-M. Lai, J. Phys. D. Appl. Phys., 2010,43 , 075104,

37 E. D. Palik, Handbook of Optical Constants of Solids, 1985

38 F. Toschi, D. Catone, P. O'Keeffe, A. Paladini, S. Turchini, J. Dagar and T. M. Brown, Adv. Funct. Mater., 2018, 28, 1707126.

39 S. D'Addato, V. Grillo, S. Altieri, S. Frabboni, F. Rossi and S. Valeri, J. Phys. Chem. C, 2011, 115, 14044-14049.

L. D. Marks, Reports Prog. Phys., 1994, 57, 603.

41 S. D'Addato, D. Pinotti, M. C. Spadaro, G. Paolicelli, V. Grillo, S. Valeri, L. Pasquali, L. Bergamini and S. Corni, Beilstein J Nanotechnol, 2015, 6, 404-413.

42 W.-H. Kim, W. J. Maeng, M.-K. Kim, J. Gatineau and H. Kim, J. Electrochem. Soc., 2011, 158, g217.

43 S. Guo, H. Arwin, S. N. Jacobsen, K. Järrendahl and U. Helmersson, J. Appl. Phys., 1995, 77, 5369-5376.

44 B. Choudhury and A. Choudhury, Mater. Chem. Phys., 2012, 131, 666-671.

45 X. Lu, D. Zheng, P. Zhang, C. Liang, P. Liu and Y. Tong, Chem. Commun., 2010, 46, 7721.

46 P. Patsalas, S. Logothetidis, L. Sygellou and S. Kennou, Phys. Rev. B - Condens. Matter Mater. Phys., 2003, 68, 035104. M. M. Khan, S. A. Ansari, D. Pradhan, D. H. Han, J. Lee and M. H. Cho, Ind. Eng. Chem. Res., 2014, 52, 9754-9763. G. V. Hartland, Phys. Chem. Chem. Phys., 2004, 6, 5263.

48 G. V. Hartland, Phys. Chem. Chem. Phys., 2004, 6, 5263. Sci., 2016, 464, 18-24.

50 L. Amidani, A. Naldoni, M. Malvestuto, M. Marelli, P. Glatzel, V. DalSanto and F. Boscherini, Angew. Chemie, 2015, 127, 5503-5506.

51 P. J. Hay, R. L. Martin, J. Uddin and G. E. Scuseria, J. Chem. Phys., 2006, 75, 1-6.

52 F. Esch, S. Fabris, L. Zhou, T. Montini, C. Africh, P. Fornasiero, G. Comelli and R. Rosei, Science (80-. )., 2005, 309, 752-755. ha formattato: Tipo di carattere: Grassetto

ha eliminato:

ha formattato: Tipo di carattere: Grassetto

ha eliminato: DOI:10.1088/0022-3727/43/7/075104 